\title{
A recurrent missense variant in HARS2 results in variable sensorineural hearing loss in three unrelated families
}

\author{
Leigh A. M. Demain ${ }^{1,2} \cdot$ Erica. H. Gerkes ${ }^{3} \cdot$ Richard J. H. Smith $^{4} \cdot$ Leslie P. Molina-Ramirez $^{1,2} \cdot$ Raymond T. O'Keefe $^{1}$. \\ William G. Newman $\mathbb{D}^{1,2}$
}

Received: 4 June 2019 / Revised: 14 November 2019 / Accepted: 2 December 2019 / Published online: 12 December 2019

(c) The Author(s) 2019. This article is published with open access

\begin{abstract}
HARS2 encodes mitochondrial histidyl-tRNA synthetase (HARS2), which links histidine to its cognate tRNA in the mitochondrial matrix. Biallelic variants in HARS2 are associated with Perrault syndrome, a rare recessive condition characterized by sensorineural hearing loss in both sexes and primary ovarian insufficiency in 46,XX females. Some individuals with Perrault syndrome have a broader phenotypic spectrum with neurological features, including ataxia and peripheral neuropathy. Here, we report a recurrent variant in HARS2 in association with sensorineural hearing loss. In affected individuals from three unrelated families, the variant $H A R S 2$ c. $1439 \mathrm{G}>\mathrm{A}$ p.(Arg480His) is present as a heterozygous variant in trans to a putative pathogenic variant. The low prevalence of the allele HARS2 c.1439G $>\mathrm{A}$ p. (Arg480His) in the general population and its presence in three families with hearing loss, confirm the pathogenicity of this variant and illustrate the presentation of Perrault syndrome as nonsyndromic hearing loss in males and prepubertal females.
\end{abstract}

\section{Introduction}

HARS2 encodes mitochondrial histidyl-tRNA synthetase (HARS2). HARS2 links histidine to its cognate tRNA in the mitochondrial matrix [1] and is an essential factor for mitochondrial translation [2]. Biallelic variants in HARS2 have been associated with Perrault syndrome, a rare autosomal recessive disease characterised by variable degrees of sensorineural hearing loss (SNHL) in both sexes and primary ovarian insufficiency (POI) in 46, XX karyotype

William G. Newman

william.newman@manchester.ac.uk

1 Division of Evolution and Genomic Sciences, School of Biological Sciences, Faculty of Biology, Medicine and Health, University of Manchester, Manchester, UK

2 NW Genomic Laboratory hub, Manchester Centre for Genomic Medicine, St Mary's Hospital, Manchester University NHS Foundation Trust, Manchester, UK

3 Department of Genetics, University of Groningen, University Medical Center Groningen, Groningen, The Netherlands

$4 \quad$ Molecular Otolaryngology and Renal Research Laboratories and the Department of Otolaryngology-Head and Neck Surgery, Carver College of Medicine, University of Iowa, Iowa City, IA, USA females $[3,4]$. In some cases of Perrault syndrome additional neurological features, including peripheral neuropathy, cerebellar ataxia, and intellectual disability have also been identified [5]. Some individuals are reported to have white matter changes noted on magnetic resonance imaging of the brain [6]. To date, biallelic variants in six causative genes have been associated with Perrault syndrome:HSD17B4 (MIM 233400) [7], HARS2 (MIM 614926) [3], LARS2 (MIM 615300) [8], CLPP (MIM 614129) [9], C10orf2 (MIM 616138) [10], and ERAL1 (MIM 607435) [6].

Previously, six unrelated families have been reported with variants in HARS2 which cause Perrault syndrome. In a large family from North America, five affected individuals were compound heterozygous for the variants HARS2 c.598C $>\mathrm{G}$ p.(Leu200Val) and c.1102G $>\mathrm{T}$ p.(Val368Leu). These variants reduced the aminoacylation activity of HARS2. All affected siblings had bilateral SNHL that varied in severity and age of onset. The three affected female siblings had ovarian dysgenesis and a 46,XX karyotype [3]. Two unrelated women with Perrault syndrome from consanguineous Moroccan families were homozygous for the same variant, HARS2 c.1010A >G p.(Tyr337Cys). Both affected individuals are from the same region in Morocco and share a haplotype consistent with a founder variant. They have a similar phenotype of profound SNHL with onset before 3 years of age and secondary amenorrhea 
presenting at 25 and 26 years of age, respectively [11]. Three unrelated families with bialleic variants in HARS2 were reported to all have rapidly progressive hearing loss [12]. In one family with SNHL, three affected individuals were compound heterozygous for the variants HARS 2 c. $172 \mathrm{~A}>\mathrm{G}$ p.(Lys58Glu) and c.448C $>\mathrm{T}$ p.(Arg150Cys). Of note the two affected females in this family were 13 and 16 years of age, respectively. The proband from the second family, a 7year-old female, was compound heterozygous for the variants HARS2 c. $448 \mathrm{C}>\mathrm{T}$ p.(Arg150Cys) and c. $980 \mathrm{G}>\mathrm{A}$ p.(Arg327Gln). The third family comprised a 32-year-old female with Perrault syndrome and the variants HARS2 c.137T $>$ A p.(Leu46Gln) and c.259C $>$ T p.(Arg87Cys) [12].

Here, we report three individuals with SNHL and previously unreported biallelic variants in HARS2. In each case, the affected individual is compound heterozygous for the variant HARS2 c.1439G $>$ A, p.(Arg480His) and a second putative pathogenic variant in HARS2.

\section{Materials and methods}

Informed consent was obtained from all individual participants included in the study. All procedures performed in studies involving human participants were in accordance with the ethical standards of the institutional and/or national research committee and with the 1964 Helsinki declaration and its later amendments or comparable ethical standards. Ethical approval for this study was granted by the National Health Service Ethics Committee (16/WA/0017) and University of Manchester. Exome sequencing for individual F1II-1 was performed by the Radboud University Medical Center, Nijmegen, The Netherlands. Before sequencing, genomic DNA fragments were enriched for exome sequences using the Agilent (Santa Clara, CA, USA) SureSelectXT Human All Exon $50 \mathrm{Mb}$ kit. WES was performed at BGI-Europe (Copenhagen, Denmark), employing an Illumina HiSeq machine (Illumina, San Diego, CA, USA). Read alignment using the Burrows Wheeler algorithm and variant calling with GATK were performed at BGI. Variants were annotated with an in-house developed annotation and prioritization pipeline. Reported variants were only confirmed with Sanger sequencing in case of low quality (GATK quality scores) of the variant. Copy number variant calling was performed using CoNIFER 0.2.0, and variant annotation was performed using an in-house developed strategy. The median coverage was $97.5 \%$ with the HiSeq system. A panel of 142 hearing loss genes was analyzed [13]. Variants were classified according to the existing guidelines from the American College of Medical Genetics and Genomics [14]. The variants in families F2 and F3 were identified by hearing loss panel (OtoSCOPE) at the Molecular Otolaryngology and Renal Research
Laboratories at the University of Iowa, a Clinical Laboratory Improvement Amendments accredited laboratory. OtoSCOPE ${ }^{\bullet}$ is a custom next-generation sequencing panel. Variants are mapped and analyzed using a custom pipeline before being confirmed by Sanger Sequencing. OtoSCOPE has a diagnostic sensitivity and specificity of $>99 \%$ [15] All variants were mapped to the transcript NM_012208.3. Prediction of variant pathogenicity was performed using the following online resources: gnomAD (http://gnomad.broa dinstitute.org/) [16], MutationTaster (http://www.mutationta ster.org/) [17], PolyPhen-2 (http://genetics.bwh.harvard. edu/pph2) [18], SIFT (http://sift.jcvi.org/) [19], ClustalOmega (https://www.ebi.ac.uk/Tools/msa/clustalo/). The variants have been submitted to the ClinVar database (https://www.ncbi.nlm.nih.gov/clinvar/), accession numbers; SCV000924703, SCV000924704, SCV000924705, and SCV000924706.

\section{Results}

\section{Clinical reports}

Family F1 is a nonconsanguineous family of European descent and comprises an affected female proband, an unaffected younger child and her unaffected parents (Fig. 1a). The proband was diagnosed with moderate bilateral SNHL at age 6 years and was fitted with hearing aids. Audiometric testing revealed a more pronounced level of hearing loss at low frequencies (Fig. 1b). An average pure tone threshold of 48.75 in both ears at frequencies $512 \mathrm{~Hz}, 1,2$, and $4 \mathrm{kHz}$ of $48.75 \mathrm{~dB}$ (HL) was reported. At last assessment, no additional clinical features were present in the proband (Table 1). No relevant family history of similar hearing loss was reported.

Compound heterozygous variants in HARS2; c. $413 \mathrm{G}>\mathrm{A}$ p.(Arg138His), inherited maternally, and c.1439G $>$ A p. (Arg480His) (NM_012208.3), inherited paternally, were identified as the likely cause of SNHL in the proband. The younger unaffected sibling was wild type at both loci.

Family F2 is a nonconsanguineous family comprising two affected male siblings and their unaffected parents (Fig. 1a). Proband F2-II-1 presented with bilateral mild-tomoderate SNHL at age 2.5 years. A mean pure tone threshold of 56.25 and $58.75 \mathrm{~dB}$ (HL) for the left ear at speech frequencies was reported. Further audiometric testing at age 9 years revealed an average pure tone threshold of $77.5 \mathrm{~dB}$ (HL) for the right ear and $71.25 \mathrm{~dB}$ (HL) for the left ear at frequencies $512 \mathrm{~Hz}, 1,2$, and $4 \mathrm{kHz}$ (Fig. 1b). In light of the hearing loss in the older sibling, audiological testing for the younger sibling was requested (F2-II-2). Mild-tomoderate bilateral SNHL was detected in this sibling at age 1. Further audiometric testing at age 6 years revealed a 
A

F1

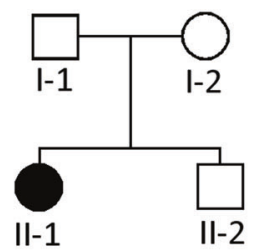

F2

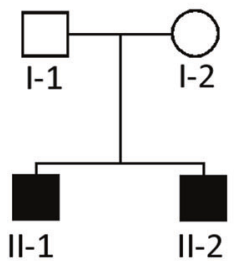

F3

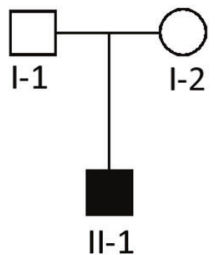

C

Human_HARS2 Human_HARS Chimpanzee

Dog

Mouse

Chicken

Xenopus

Tetraodon

Zebrafish

C_Elegans

S_cerevisiae

E_Coli_HisRS

Human_HARS2

Human_HARS

Chimpanzee

Dog

Rat

Mouse

Chicken

Xenopus

Tetraodon

Zebrafish

c Elegans

S_cerevisiae

E_coli_hisRs
B

F1 II-1 aged 6 years

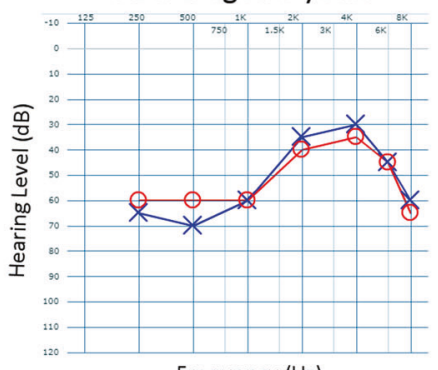

Frequency $(\mathrm{Hz})$

F2 II-1 aged 9 years

F2 II-2 aged 6 years
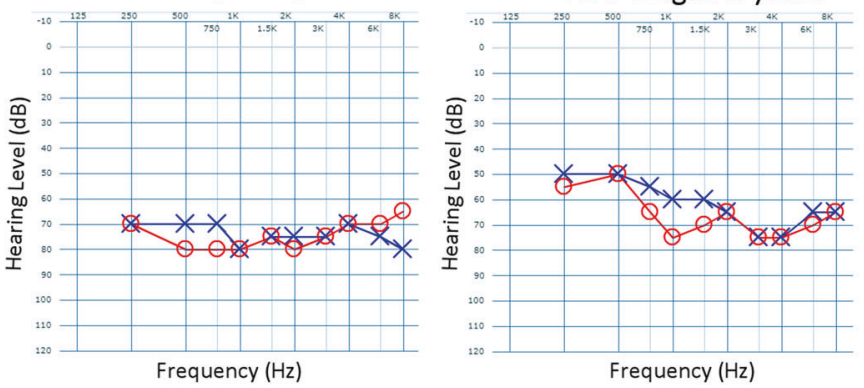

F3 II-1 aged 4 years

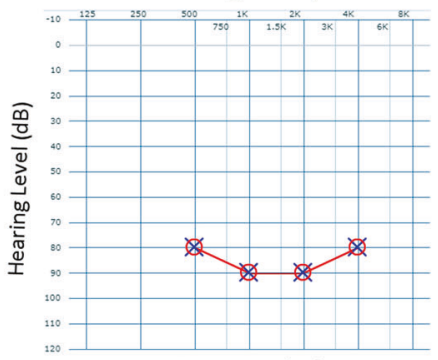

Frequency $(\mathrm{Hz})$

R138

TLTEKYGE---DS-GLMYDLKDQGGELLSLRYDLTVPFARYLAMN---KVKKMKRYHVGK 155

TLMGKYGE---DS-KLIYDLKDQGGELLSLRYDLTVPFARYLAMN---KLTNIKRYHIAK 154

TLTEKYGE---DS-GLMYDLKDQGGELLSLRYDLTVPFARYLAMN---KVKKMKRYHVGK 155 MLTEKYGE---DS-GLIYDLKDQGGELLSLRYDLTVPFARYLAMN---KVKRMKRYQVGK 156 MLTEKYED---NF-GLMYDLEDQGGELLSLRYDLTVPFARYLAMN---KLKRMKRYQVGK 156 MLTEKYED---NF-GLMYDLKDQGGELLSLRYDLTVPFARYLAMN---KLKKMKRYOVGK 154 TLMEKYGD---NS-KLIYELODQGGELLALRYDLTVPFARYLAMN---KITNIKRYHIAK 85 TLMEKYGD---NS-KLIYELQDQGGELLALRYDLTVPFARYLAMN---KITNIKRYHIAK 85 $\begin{array}{lll} & \\ \text { ISKATFNH---CL-LIIFFFQMTFYQLHTVRVRVKVPFARYLAMN---KITNIKRYHIAK } & 156\end{array}$ TLTGKYGE---DS-KLIYDLKDQGGELLSLRYDLTVPFARYLAMN---KITNIKRYHIAK 161 VLMGKYGE---EGGKLVYDLQDQGGELLSLRYDLTVPFARYLAMN---KITNITRYQIAK 164 ILAGKYGE---DS-KLIYNLEDQGGELCSLRYDLTVPFARYVAMN---NIQSIKRYHIAK 153 LFKRAIGEVTDVVEKEMYTFEDRNGDSLTLRPEGTAGCVRAGIEHGLLYNQEQRLWYIGP 110

R480

LVVIIGEQELKEGVIKIRSVASREEV----AIKRENFVAEIQKRLSES---------- 506 LVAIIGEQELKDGVIKLRSVTSREEV----DVRREDLVEEIKRRTGQPLCIC------ 509 LVVIIGEQELKEGVIKIRSVASREEV----SGGSRNRRDEVFLPFPDSVRNMHLLAEKLS 518 LVVIIGEQELKEGVIKLRSVASREEV----AIKRENLVAEIQKKLSES----------- 506

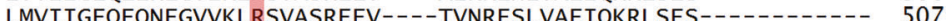
LMVIIGEQERNEGVIKLRSVASREEV LVAIIGEQELRDGVVLLDATREEV - DIPREEAEIRR LVAIIGEQELRDGVVKLRDAATREEV-DDIPREELAAEIRRRLETCTTGNPAAPQPLP 448 LVAIIGEQELKDGVVKLRVVATREEV----DVARQNLVEEIHKRTQIFSTGC------- 393 LVAILGEQELKNGVVKLRDVATRDEV----DVSRAELIAEIKRRTSEA---------- 517 LVAILGEQELKDGVVKLRNVASREEV----DVPRAELVDEVKKRTS----------- 520

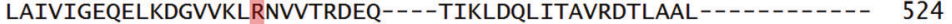
IAVILGKEEYLEGKLRVKRLGQEFADDDGELVSAADIVPIVOEKLSQIHEDGLNEVTRLI 543 VAVVLGESEVANGTAVVKDLRSGEQT----AVAQDSVAAHLRTLLG-_-.---.-- 424 mean pure tone threshold of $60 \mathrm{~dB}$ (HL) for the right ear and $58.75 \mathrm{~dB}(\mathrm{HL})$ for the left ear at frequencies $512 \mathrm{~Hz}, 1$, 2 , and $4 \mathrm{kHz}$ (Fig. 1b).
Due to progression of the hearing loss, both siblings were reported to have undergone unilateral cochlear implantation. Both affected siblings have soft neurological features, for 
Fig. 1 Pedigree, air conduction thresholds, and protein sequence alignment in individuals with HARS2 variants listed in Table 1. a Pedigrees for families F1-F3. Filled icons indicate affected individuals. b Audiograms from affected individuals in families F1, F2, and F3. All affected individuals have bilateral sensorineural across all frequencies. Proband F1-II-1 shows lower air conduction thresholds in lower frequencies. Circles represent the right ear and crosses the left ear, $\mathrm{dB}$ decibels. Audiograms were created using the AudGen online tool (version 0.71) (http://audsim.com/audgen/). c The conservation of histidyl-tRNA synthetase across multiple species. The variant residues p.Arg 138 and p.Arg480 are shown in the red boxes. Numbering relates to the human HARS2 protein (GenPept: NP_036340.1). Sequences for each species are as follows; chimpanzee UniProt: A0A2I3RUB6, dog UniProt:F6XKV4, rat GenPept:NP_001014034.2, mouse GenPept:NP_542367.1, chicken UniProt:A0A1D5P330, xenopus UniProt: F7A0W8, zebrafish GenPept:NP_001289185.1, tetraodon UniProt: H3CFH8, C. elegans GenPept:NP_001023373.2, S. cerevisiae GenPept:NP_015358.1, and E. coli HisRS UniProt: P60906.

example, one has difficulty with fine motor movements, and the other has tightness in the muscles of his lower limbs. Neurological phenotypes are a well-recognized feature of Perrault syndrome; however, there is no concordance in these features between the brothers. Both siblings have had problems with tooth growth (Table 1). The compound heterozygous variants HARS2; c.828delTinsGTATCCCTAGTATTTCTACTA p.(Gly277TyrfsTer3) and c.1439G >A, p.(Arg480His) (NM_012208.3) were identified as the likely cause of SNHL in the affected siblings.

Family F3 is a nonconsanguineous family comprising the male proband (F3-II-1) and his unaffected parents (Fig. 1a). Bilateral profound SNHL was detected in this proband at 23 months of age. Sedated auditory brainstem response reported a mean pure tone threshold of $85 \mathrm{~dB}$ (HL) for both ears (Fig. 1b). Of note during testing using reversed polarity air conduction click a reversal of the cochlear microphonic was seen in both ears, suggestive of auditory neuropathy. At his last assessment, the proband was 4 years and had no additional clinical features (Table 1). The proband is currently under assessment for cochlear implantation. The variants HARS2; c.72C>A, p.(Cys24Ter) and c.1439G >A p. (Arg480His) (NM_012208.3) were identified as the likely cause of SNHL in the proband.

\section{Variants and predicted consequences}

Human HARS2 likely functions as a homodimer. It contains predicted domains for histidine binding, dimer interaction, and tRNA binding [20]. HARS2 (NP_036340.1) shares $\sim 73 \%$ sequence homology with nuclear HARS (NP_002100.2), from which it primarily differs at the N-terminus, and shares $\sim 23 \%$ sequence homology with the E.coli orthologue HisRS (P60906).

The variant HARS2 p.(Arg138His) in individual F1-II-1 is present as a heterozygous variant in eight individuals of 141,443 sequenced (minor allele frequency, 0.000028) in
gnomAD and has never been seen as a homozygous variant. This low-carrier frequency is consistent with a variant causative of rare autosomal recessive disease. HARS2 p.(Arg138His) is also predicted to be deleterious by multiple in silico analysis tools. The residue HARS2 Arg138 is an almost invariant residue conserved in both human HARS and the E.coli orthologue HisRS (Fig. 1c). Arg138 is situated in the predicted dimer interface region of HARS2. In E.coliHisRS, residue Arg90 (equivalent to residue Arg138 in HARS2) is located in the dimer interface region of the protein. E.coli HisRS Arg90 forms salt bridges with residues Asp13 and Glu47 of the other monomer in the dimer complex. These interactions both facilitate dimer formation and shape the active site [20]. The residues equivalent to Asp13 and Glu47 in human HARS2 are also conserved (Asp65 and Glu99 respectively). The substitution of arginine to histidine at residue 138 may interfere with salt bridge formation and subsequently dimer interaction and active site confirmation of HARS2.

The second variant in individual F1-II-1 HARS2 p.(Arg480His) is reported as a heterozygous variant in 21 of 141,421 individuals (minor allele frequency 0.000074) in gnomAD and never as a homozygous variant. HARS2 p.(Arg480His) is also predicted to be deleterious by multiple in silico analysis tools. The residue Arg480 is located in the C-terminal domain of HARS2, which is predicted as important for tRNA recognition and binding [20]. Residue Arg480 is well conserved but not as strictly invariant as the residue Arg138 (Fig. 1c). The effect of the substitution of Arginine for histidine at residue 480 is unclear, but it may disrupt recognition or binding of mitochondrial tRNA ${ }^{\text {His }}$.

In the affected individuals in families F2 and F3 the variant p.(Arg480His) is in trans with a loss-of-function variant, p.(Gly277TyrfsTer3) and p.(Cys24Ter), respectively. The transcripts for both of the loss-of-function variants are predicted to be subjected to nonsense mediated decay. Neither variant was present in gnomAD as either a heterozygous or homozygous variant. No homozygous loss-of-function variants are reported in HARS2 in gnomAD.

\section{Discussion}

Here, we report the variant HARS2 c.1439G $>$ A p.(Arg480His) in three unrelated families with prelingual onset, bilateral symmetric progressive SNHL. In each family the variant was in trans to a second putative pathogenic variant in HARS2. The identification of this rare missense variant in three unrelated individuals with SNHL means that it can be classified as moderate evidence of pathogenicity according to the American College of Medical Genetics and Genomics guidance on variant 


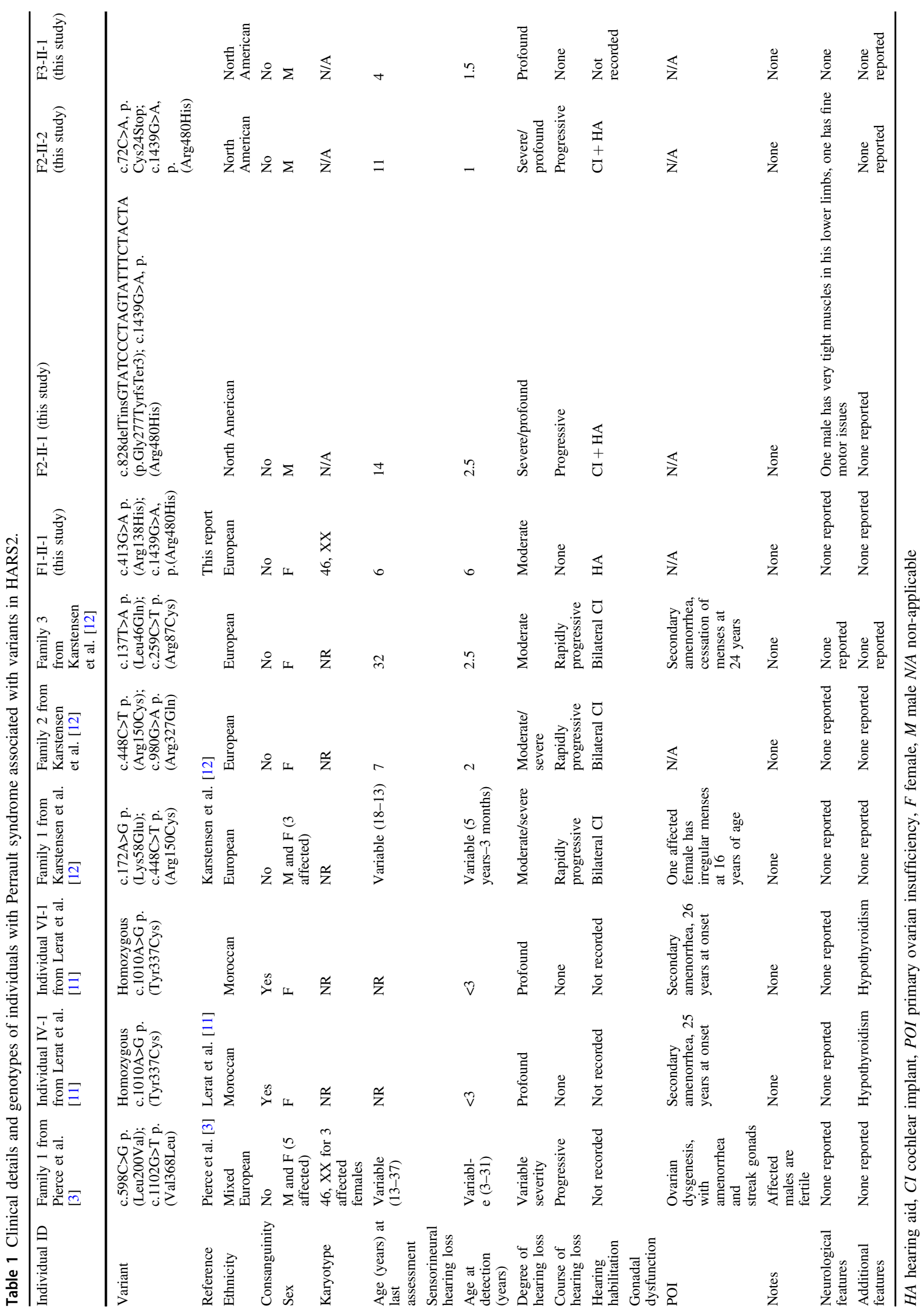


pathogenicity [14]. The report of these families increases the number of described families with HARS2 variants and either Perrault syndrome or SNHL to nine.

In the proband from family $\mathrm{F} 1$ the variant in trans to HARS2 p.Arg480His may interrupt the dimerization and active site confirmation of the HARS2 homodimer. Given the conservation and function of p.Arg138 as well as the in silico predictions and the minor allele frequency it is likely that the substitution p.Arg138His is deleterious. In affected individuals from families $\mathrm{F} 2$ and F3 the variant p.(Arg480His) is in trans to a loss-of-function variant. No cases of biallelic loss-of-function variants in any of the six genes associated with Perrault syndrome have been reported to date [21]. This lack of biallelic loss-of-function variants likely indicates that HARS2, along with the other Perrault syndrome associated genes, is essential and that complete loss-of-function would result in lethality. Of note, the loss of the HARS2 yeast orthologue, HST1, is lethal [22]. If homozygous loss of HARS2 is lethal only combinations of hypomorphic alleles in trans with loss-of-function alleles, or biallelic hypomorphic alleles can be expected to result in a phenotype.

Of note, the hearing loss in the individual with two missense (putative hypomorphic) HARS2 variants is milder (especially at higher frequencies) than in the other two families, where a missense (hypomorphic) variant is in trans with a predicted loss-of-function variant. Further follow-up of these individuals and ascertainment of additional cases will determine if this genotype-phenotype association represents a robust observation.

Currently, there appears to be no link between the variants in HARS2 reported to be causative of Perrault syndrome and their location in the HARS2 protein. The reported pathogenic variants in HARS2 are located in multiple domains with no specific domain linked to dysfunction. In contrast, variants in CLPP linked to Perrault syndrome are clustered mainly in a single 20 residue region of the CLPP protein [21].

In the families reported here a diagnosis of SNHL revealed variants in a Perrault syndrome related gene, which would not have been suspected based on clinical presentation alone. All affected individuals are either male or prepubertal and as such would not present with POI, a cardinal feature of Perrault syndrome. In the case of family F1 the female proband is prepubertal and will be monitored for POI. It is possible that hypomorphic variants in Perrault syndrome genes may cause milder presentation of hearing loss or POI only and therefore the individuals from F1 or F3 may have nonsyndromic SNHL [21]. It is less likely that the neurological features reported in family F2 can be attributed to the HARS2 variants as they are not concordant in the affected siblings. Further follow-up and evaluation of additional individuals with
HARS2 pathogenic variants will be required to establish any neurological phenotype. Neurological presentations are commonly associated with Perrault syndrome and although no phenotype genotype links have yet been established for $H A R S 2$, all individuals with disease associated HARS2 variants should have a full clinical neurological assessment. As panel and exome testing becomes more commonplace for etiological diagnosis in hearing loss, we expect more cases of HARS2-associated Perrault syndrome will be recognized. Timely confirmation of etiological diagnosis and subsequent prediction of profound severity in individuals with HARS2 variants could help inform the identification of cases that may benefit from referral to cochlear implantation services and closer audiological surveillance [23].

Acknowledgements We thank the families for their participation in this study. The study was supported in part by Action Medical Research (GN2494), the NIHR Manchester Biomedical Research Centre (IS-BRC1215-20007), National Institute on Deafness and Other Communication Disorders (R01 DC012049) (RJHS), and National Council of Science and Technology in Mexico (CONACyT) (LPMR).

\section{Compliance with ethical standards}

Conflict of interest The authors declare that they have no conflict of interest.

Publisher's note Springer Nature remains neutral with regard to jurisdictional claims in published maps and institutional affiliations.

Open Access This article is licensed under a Creative Commons Attribution 4.0 International License, which permits use, sharing, adaptation, distribution and reproduction in any medium or format, as long as you give appropriate credit to the original author(s) and the source, provide a link to the Creative Commons license, and indicate if changes were made. The images or other third party material in this article are included in the article's Creative Commons license, unless indicated otherwise in a credit line to the material. If material is not included in the article's Creative Commons license and your intended use is not permitted by statutory regulation or exceeds the permitted use, you will need to obtain permission directly from the copyright holder. To view a copy of this license, visit http://creativecommons. org/licenses/by/4.0/.

\section{References}

1. O'Hanlon TP, Raben N, Miller FW. A novel gene oriented in a head-to-head configuration with the human histidyl-tRNA synthetase (HRS) gene encodes an mRNA that predicts a polypeptide homologous to HRS. Biochem Biophys Res Commun. 1995;210:556-66.

2. Diodato D, Ghezzi D, Tiranti V. The mitochondrial aminoacyl tRNA synthetases: genes and syndromes. Int $\mathrm{J}$ Cell Biol. 2014;2014:787956.

3. Pierce SB, Chisholm KM, Lynch ED, Lee MK, Walsh T, Opitz JM, et al. Mutations in mitochondrial histidyl tRNA synthetase HARS2 cause ovarian dysgenesis and sensorineural hearing loss of Perrault syndrome. Proc Natl Acad Sci USA. 2011;108:6543-8. 
4. Pallister PD, Opitz JM. The Perrault syndrome: autosomal recessive ovarian dysgenesis with facultative, non-sex-limited sensorineural deafness. Am J Med Genet. 1979;4:239-46.

5. Gottschalk ME, Coker SB, Fox LA. Neurologic anomalies of Perrault syndrome. Am J Med Genet. 1996;65:274-6.

6. Theunissen TE, Szklarczyk R, Gerards M, Hellebrekers DM, Mulder-Den Hartog EN, Vanoevelen J, et al. Specific MRI abnormalities reveal severe Perrault syndrome due to CLPP defects. Front Neurol. 2016;7:203.

7. Pierce SB, Walsh T, Chisholm KM, Lee MK, Thornton AM, Fiumara A, et al. Mutations in the DBP-deficiency protein HSD17B4 cause ovarian dysgenesis, hearing loss, and ataxia of Perrault syndrome. Am J Hum Genet. 2010;87:282-8.

8. Pierce SB, Gersak K, Michaelson-Cohen R, Walsh T, Lee MK, Malach D, et al. Mutations in LARS2, encoding mitochondrial leucyltRNA synthetase, lead to premature ovarian failure and hearing loss in Perrault syndrome. Am J Hum Genet. 2013;92:614-20.

9. Jenkinson EM, Rehman AU, Walsh T, Clayton-Smith J, Lee K, Morell RJ, et al. Perrault syndrome is caused by recessive mutations in CLPP, encoding a mitochondrial ATP-dependent chambered protease. Am J Hum Genet. 2013;92:605-13.

10. Morino H, Pierce SB, Matsuda Y, Walsh T, Ohsawa R, Newby M, et al. Mutations in twinkle primase-helicase cause Perrault syndrome with neurologic features. Neurology. 2014;83:2054-61.

11. Lerat J, Jonard L, Loundon N, Christin-Maitre S, Lacombe D, Goizet C, et al. An application of NGS for molecular investigations in Perrault syndrome: study of 14 families and review of the literature. Hum Mutat. 2016;37:1354-62.

12. Karstensen HG, Rendtorff ND, Hindbaek LS, Colombo R, Stein A, Birkebaek NH, et al. Novel HARS2 missense variants identified in individuals with sensorineural hearing impairment and Perrault syndrome. Eur J Med Genet. 2019;103733.

13. Zazo Seco C, Wesdorp M, Feenstra I, Pfundt R, Hehir-Kwa JY, Lelieveld $\mathrm{SH}$, et al. The diagnostic yield of whole-exome sequencing targeting a gene panel for hearing impairment in The Netherlands. Eur J Hum Genet. 2017;25:308-14.

14. Richards S, Aziz N, Bale S, Bick D, Das S, Gastier-Foster J, et al. Standards and guidelines for the interpretation of sequence variants: a joint consensus recommendation of the American College of Medical Genetics and Genomics and the Association for Molecular Pathology. Genet Med. 2015;17:405-24.

15. Shearer AE, DeLuca AP, Hildebrand MS, Taylor KR, Gurrola J 2nd, Scherer S, et al. Comprehensive genetic testing for hereditary hearing loss using massively parallel sequencing. Proc Natl Acad Sci USA. 2010;107:21104-9.

16. Lek M, Karczewski KJ, Minikel EV, Samocha KE, Banks E, Fennell T, et al. Analysis of protein-coding genetic variation in 60,706 humans. Nature. 2016;536:285-91.

17. Schwarz JM, Cooper DN, Schuelke M, Seelow D. MutationTaster2: mutation prediction for the deep-sequencing age. Nat Methods. 2014;11:361-2.

18. Adzhubei IA, Schmidt S, Peshkin L, Ramensky VE, Gerasimova A, Bork P, et al. A method and server for predicting damaging missense mutations. Nat Methods. 2010;7:248-9.

19. Kumar P, Henikoff S, Ng PC. Predicting the effects of coding non-synonymous variants on protein function using the SIFT algorithm. Nat Protoc. 2009;4:1073-81.

20. Arnez JG, Harris DC, Mitschler A, Rees B, Francklyn CS, Moras D. Crystal structure of histidyl-tRNA synthetase from Escherichia coli complexed with histidyl-adenylate. EMBO J. 1995;14:4143-55.

21. Newman WG, Friedman TB, Conway GS, Demain LAM. Perrault Syndrome. In: Adam MP, et al. editors. GeneReviews(R). Seattle: WA; 2014.

22. Natsoulis G, Hilger F, Fink GR. The HTS1 gene encodes both the cytoplasmic and mitochondrial histidine tRNA synthetases of $\mathrm{S}$. cerevisiae. Cell. 1986;46:235-43.

23. Molina-Ramirez LP, Bruce IA, Black GCM Cochlear implantation in the era of genomic medicine. Cochlear Implant Int. 2019;1-4. 